\title{
O COMPORTAMENTO DO ELÉTRON: UMA ANÁLISE DO EFEITO COMPTON E DA RELAÇÃO DE BROGLIE
}

\author{
Nelson H. Morgon* \\ Instituto de Química, Universidade Estadual de Campinas, CP 6154, 13084-862 Campinas - SP, Brasil
}

Recebido em 29/3/07; aceito em 29/2/08; publicado na web em 24/9/08

\begin{abstract}
THE BEHAVIOR OF THE ELECTRON: AN ANALYSIS OF THE COMPTON EFFECT AND THE DE BROGLIE'S RELATION. The De Broglie's relation was a fundamental step in the development of a wave nature for matter. Therefore, we have examined it from the perspective of the new atomic theory. This relation makes successful predictions of the stable energy levels for electrons orbiting a nucleus. The formulation of the de Broglie's relation is a fundamental application of the theory of wave-particle duality for a material particle. In this work, the direct demonstration employing the equations $E=m c^{2}$ and $E=h c / \lambda$, was avoided. We provide a complete analysis of this relation considering features of the special theory of relativity.
\end{abstract}

Keywords: De Broglie's relation; relativistic effects; modern atomic theory.

\section{INTRODUÇÃO}

Arthur H. Compton, em 1922, observou o aumento do comprimento de onda de raios $\mathrm{X}$, devido ao espalhamento da radiação incidente sobre elétrons livres. Este efeito, conhecido atualmente como efeito Compton, ilustra claramente o conceito de partícula para a radiação eletromagnética. Por esta descoberta Compton ganhou o Prêmio Nobel de Física em 1927. Esta observação é bastante interessante, pois se a radiação eletromagnética possui características de luz e partícula, a princípio deveria se esperar que partículas reais também pudessem ser descritas em termos de ondas. Em 1924 De Broglie, combinando as idéias de Planck e Einstein, estabeleceu uma relação entre massa e frequiência, comprovada experimentalmente em 1927 por Davisson e Germer. Em 1929 Louis de Broglie, pela descoberta de características de onda de elétrons, ganhou o Nobel de Física, assim como Davisson em 1937, "por suas descobertas experimentais da difração de elétrons por cristais", compartilhou o mesmo prêmio com George Paget Thomson. ${ }^{1}$

A partir desta nova abordagem para a descrição, tanto de partículas como de radiação, foi possível estabelecer um novo modelo de átomo, expresso em 1926 pela equação de Schrödinger, base para o modelo atual de estrutura eletrônica. Erwin Schrödinger e Paul A. M. Dirac dividiram o Nobel em Física de 1933, pela descoberta e elaboração desta nova teoria atômica. ${ }^{1}$

A apresentação de uma abordagem mais detalhada sobre o efeito Compton e a relação de De Broglie, envolvendo a descrição fenomenológica do comportamento do elétron, é o objetivo deste trabalho. A motivação para isto foi adquirida em sala de aula, nas disciplinas de graduação oferecidas pelo Instituto de Química aos alunos do curso de química e de outros cursos da UNICAMP, onde conceitos introdutórios de estrutura eletrônica são apresentados.

Em muitas situações, tem sido observada alguma confusão no tratamento matemático para derivar a relação onda-partícula, e expressá-la em termos do momento da partícula $(p)$ e do comprimento de onda $(\lambda)$ associado a ela, representada por

$p=\frac{h}{\lambda}$

*e-mail: morgon@iqm.unicamp.br
A importância da obtenção desta expressão, vai além de uma simples manipulação matemática das Equações

$E=m c^{2}$ e $E=h v$

A questão não é fazer apenas a igualdade destas equações

$m c^{2}=\frac{h c}{\lambda} \Rightarrow m c=\frac{h}{\lambda}$

para $v=c / \lambda$ e "assumindo-se" $p=m c$, originando-se a Equação 1. Este tratamento simplificado, em algumas situações pode até ser feito, porém com alguma restrição.

De modo geral, os livros textos tangenciam na demonstração das relações de Compton e de De Broglie, para os fenômenos corpuscular (da luz) e ondulatório (do elétron), respectivamente. Vale destacar, que não se trata de uma crítica pura e simples, pois muitas vezes a abordagem mais rigorosa foge do foco, do escopo do texto apresentado nestes livros, ou mesmo da apresentação de conhecimentos básicos de relatividade. Algumas abordagens, presentes em textos selecionados em níveis diferentes (Química Geral e Química Quântica), são mostradas a seguir, enfatizando-se que, enquanto na primeira a idéia é apresentada mais em termos de modelo atômico, na segunda há um rigor maior, já que o tratamento aprofundado se dá em termos matemáticos explorando conteúdos de Cálculo Diferencial e Integral. Para uma abordagem histórica, sobre o início do desenvolvimento do modelo atômico atual, recomenda-se a leitura dos trabalhos de Rosa e Martins, ${ }^{2,3}$ que exploram o conceito onda-partícula, do início do século XX até o surgimento da teoria de Louis de Broglie.

Nos livros introdutórios de Química Geral são comuns explicações bem sucintas como:

- ...No início deste século as, hoje familiares, relações desenvolvidas por Einstein $\left(E=m c^{2}\right)$ e Planck $(E=h v)$ já eram conhecidas ... De Broglie igualou estas duas equações, obtendo a seguinte relação: $m c^{2}=h v$. É importante observarmos aqui que não obtivemos esta relação simplesmente a partir da igualdade de duas relações envolvendo energia $;^{4}$ - ...Em 1925, De Broglie propôs que um elétron livre de massa $m$, que se move com uma velocidade $v$, deve ter um 
comprimento de onda associado, dado pela equação: $\lambda=h /$ $m v$. Essa idéia era revolucionária porque ligou as propriedades de partícula do elétron ( $m$ e v) a uma propriedade de ondas $(\lambda) ;^{5}$ ou

- ...De Broglie sugeriu que o elétron, em seu movimento ao redor do núcleo, tinha associado a ele um comprimento de onda particular. Ele propôs que o comprimento de onda característico do elétron ou qualquer outra partícula depende de sua massa, m, e de sua velocidade, v. De Broglie usou o termo ondas de matéria para descrever as características ondulatórias das partículas materiais. ${ }^{6}$

Em alguns livros de Físico-Química também notamos um tratamento superficial sobre o assunto:

- ...Podemos entender a hipótese de De Broglie equacionando a expressão da energia a partir da relação da relatividade especial e da teoria quântica: $E=m c^{2} e E=h v$... De Broglie sugeriu que esta relação se aplicava às partículas, para as quais o momento é igual à massa vezes a velocidade ( $p=$ $m v)^{7}$;

-... Some progress towards coordinating these properties had already been made by Louis de Broglie when, in 1924, he had suggested on theoretical grounds that any particle, not only photons, traveling with a momentum $p$ should have (in some sense) a wavelength given by the De Broglie's relation: $\lambda=h / p ;^{8}$ ou

- ... De Broglie argued that both light and matter obey the Equation 1. Because the momentum of a particle is given by $m v$, this equation predicts that a particle of mass $m$ moving with a velocity $v$ will have a De Broglie wavelength given by $\lambda=h / m v^{9}$

E até mesmo em alguns textos introdutórios de Química Quântica observam-se deficiências:

- ... Antes de terem sido realizadas as primeiras experiências de difração de elétrons, De Broglie já havia proposto em sua tese de doutoramento (1924) a relação: $\lambda p=h ;^{10}$

- ... Hence Louis de Broglie suggested in 1923 that the motion of electrons might have a wave aspect; that an electron of mass $m$ and speed $v$ would have a wavelength $\lambda=h / m v$ $=h / p-E q .(1.4)$, associated with it, where $p$ is the linear momentum. De Broglie arrived at Eq. (1.4) by reasoning in analogy with photons. The energy of any particle (including a photon) can be expressed, according to Einstein's special theory of relativity, as $E=m c^{2}$, where $c$ is the speed of light and $m$ is the particle's relativistic mass (not its rest mass). Using $E_{\text {photon }}=h v$, we get $m c^{2}=h v=h c / \lambda$ and $\lambda=h / m c=$ $h / p$ for a photon traveling at speed c. The Eq. (1.4) is the corresponding equation for an electron; ${ }^{11}$ ou

- Em 1924, Louis de Broglie associava, através do estudo da relatividade de Einstein, o comprimento de onda com uma partícula, $\lambda=h / p$, mas de antemão já se sabia que a luz poderia se comportar como partícula. ${ }^{12}$

Como pôde ser observado nos excertos extraídos dos livros citados, a abordagem adotada é bastante superficial, mesmo em livros textos destinados a alunos mais avançados nos cursos de graduação ou pós-graduação. Assim, neste trabalho apresentaremos uma abordagem um pouco mais detalhada da obtenção das expressões, que evidenciam a natureza de partícula da luz (efeito Compton) e a natureza de onda do elétron (relação de De Broglie), embora como assinala Richard Feynman (Prêmio Nobel de Física de 1965) ${ }^{11}$ em The Relation of Wave and Particle Viewpoints, ${ }^{13} \mathrm{o}$ elétron não deve ser considerado nem sob o ponto de vista de onda, nem de partícula.

\section{Transformada de Lorentz}

No final do século XIX, houve um interesse crescente em estudos de fenômenos de eletricidade, eletromagnetismo e luz, que culminaram nas equações de Maxwell para um sistema movendo-se uniformemente. No entanto, posteriormente verificou-se que tais equações pareciam não obedecer ao princípio da relatividade de Einstein-Poincaré, definido adiante. ${ }^{13}$ Assim, elas poderiam estar erradas e deveriam ser mudadas, de modo que sob a transformada de Galileu este princípio pudesse ser satisfeito. Este tipo de transformação pode ser representada de modo bem simplificado, considerando-se o sistema de coordenadas $(x, y, z, t)$ definido inicialmente pelo conjunto de variáveis espaciais $(x, y, z)$ e temporal $(t)$, e posteriormente como $\left(x^{\prime}\right.$, $\left.y^{\prime}, z^{\prime}, t^{\prime}\right)$ à velocidade constante $v$ ao longo do eixo $x$, mantendo-se $y^{\prime}=y$ e $z^{\prime}=z$. Assim, tem-se

$$
\begin{aligned}
& \mathrm{x}=\mathrm{x}^{\prime}+v \mathrm{t} \\
& \mathrm{y}=\mathrm{y}^{\prime} \\
& \mathrm{z}=\mathrm{z}^{\prime} \\
& \mathrm{t}=\mathrm{t}^{\prime}
\end{aligned}
$$

Como dito anteriormente, as Equações de Maxwell, neste tipo de transformação de coordenadas, levavam ao surgimento de novos termos, que por sua vez levavam a previsões de novos fenômenos elétricos, que não eram observados experimentalmente. Deste modo, abandonou-se esta tentativa de transformação. ${ }^{13}$ Neste meio tempo, H. A. Lorentz (Prêmio Nobel de Física de 1902) ${ }^{1}$ aplicou as seguintes substituições nas Equações de Maxwell

$$
\begin{aligned}
& x=\left(x^{\prime}+v t^{\prime}\right) \gamma \\
& y=y^{\prime} \\
& z=z^{\prime} \\
& t=\left(t^{\prime}+\frac{v x^{\prime}}{c^{2}}\right) \gamma
\end{aligned}
$$

onde $c$ é a velocidade da luz no vácuo e $\gamma$ é conhecido por fator (ou termo) de Lorentz definido por

$$
\gamma=\frac{1}{\sqrt{1-\frac{v^{2}}{c^{2}}}}
$$

Ele notou que elas permaneceram da mesma forma sob estas transformações. As equações acima ficaram conhecidas como transformada de Lorentz. Posteriormente Einstein, seguindo sugestão formulada previamente por Poincaré, generalizou esta observação propondo que, todas as leis da física deveriam de tal modo, permanecer inalteradas sob uma transformada de Lorentz. Em outras palavras, não apenas as leis da eletrodinâmica (verificada por Lorentz) em particular, mas as leis da mecânica de um modo geral. Em situações ordinárias, onde $\mathrm{v} \ll c, \frac{\mathrm{v}^{2}}{c^{2}} \approx 0$ e $\gamma$ aproxima-se da unidade, levando-se à transformada galileana. Para uma descrição mais detalhada deste tópico recomenda-se uma leitura do capítulo 15 da ref. 13.

\section{Energia e momento relativísticos}

Com base na transformada de Lorentz, podem ser deduzidas 
expressões para outras grandezas, como massa $\left(m=m_{0} \gamma\right)$ e frequiência de onda $\left(v=v_{0} \gamma\right)$. Grandezas estas, medidas considerando-se o referencial $S$ em movimento relativo a um referencial $S_{0}$ (em repouso). Novamente, no caso onde $\mathrm{v} \ll c$, as grandezas $m$ tornam-se $m_{0} \mathrm{e} v$, $\mathrm{v}_{0}$, respectivamente. ${ }^{14}$

Diferentemente das situações consideradas "cotidianas", bem descritas pela transformada de Galileu, o comportamento de sistemas microscópicos, principalmente a altas velocidades, é bastante singular, sendo então necessário o uso da transformada de Lorentz. Vale salientar que existem muitas situações microscópicas que ocorrem a "velocidades" baixas.

O comportamento do elétron livre em movimento pode ser aproximado pela descrição da mecânica clássica, e sua energia expressa em função da sua massa de repouso $\left(m_{0}\right)$ e de sua velocidade $(v)$, ou seja, descrita apenas pela componente cinética. No entanto, existem outras situações distintas, onde o elétron se comporta de modo "não convencional" como, por exemplo, quando ele está sujeito a um campo, descrito por um poço de potencial (modelo da partícula na caixa), em ambientes atômico ou molecular, etc, tendo deste modo comportamento mecânico-quântico (comportamento de onda e partícula); ou quando ele apresenta $\mathrm{v} \approx c$, onde há necessidade de componentes relativísticas para uma descrição mais precisa.

Segundo a teoria da relatividade, a massa relativística efetiva da partícula (no nosso caso o elétron) a $\mathrm{v} \approx c$, é dada por

$m=m_{0} \gamma$

Como a partícula move-se a uma velocidade $v$, é possível reescrever a Equação 5 em termos de seu momento linear' ${ }^{15,17}$

$p=m \vee m_{0} \vee \gamma$

Expandindo-se $\gamma$ a partir da Equação 4

$$
p=\frac{m_{0} v}{\sqrt{1-\frac{v^{2}}{\mathrm{c}^{2}}}}
$$

Elevando-se ao quadrado a Equação 7 e multiplicando-se ambos os lados por $c^{2}$, obtém-se

$$
p^{2} c^{2}=\frac{m_{0}^{2} v^{2}}{1-\frac{v^{2}}{c^{2}}} c^{2} \quad \text { ou } \quad p^{2} c^{2}=\frac{m_{0}^{2} c^{4} \frac{v^{2}}{c^{2}}}{1-\frac{v^{2}}{c^{2}}} \quad m_{0}^{2} c^{4} \frac{v^{2}}{c^{2}}
$$

Adicionando-se e subtraindo-se o termo $\frac{m_{0}^{2} c^{4} \frac{v^{2}}{c^{2}}}{v^{2}}$ no lado direito da Equação 8, tem-se

$$
p^{2} c^{2}=\frac{m_{0}^{2} c^{4} \frac{v^{2}}{c^{2}}}{1-\frac{v^{2}}{c^{2}}}+\frac{m_{0}^{2} c^{4}}{1-\frac{v^{2}}{c^{2}}}-\frac{m_{0}^{2} c^{4}}{1-\frac{v^{2}}{c^{2}}}
$$

Rearranjando-se a Equação 9 de modo que o termo $m_{0}^{2} c^{4}$ fique em evidência, dá como resultado a expressão

$$
p^{2} c^{2}=\frac{m_{0}^{2} c^{4}\left(\frac{v^{2}}{c^{2}}-1\right)}{1-\frac{v^{2}}{c^{2}}}+\frac{m_{0}^{2} c^{4}}{1-\frac{v^{2}}{c^{2}}}
$$

Substituindo-se a expressão de $\gamma$ - Equação 4, na Equação 10, obtém-se

$$
p^{2} c^{2}=-\frac{m_{0}^{2} c^{4}\left(\frac{v^{2}}{c^{2}}-1\right)}{\frac{v^{2}}{c^{2}}-1}+m_{0}^{2} c^{4} \gamma^{2}
$$

Finalmente, fazendo-se uso da substituição de $m_{0} \gamma$ por $m$ dada pela Equação 5, tem-se

$$
p^{2} c^{2}=-m_{0}^{2} c^{4}+\frac{m^{2}}{\gamma^{2}} c^{4} \gamma^{2}
$$

que leva à expressão ${ }^{18}$

$$
p c=\sqrt{\left(m c^{2}\right)^{2}-m_{0}^{2} c^{4}}
$$

O termo $m c^{2}$ está relacionado à expressão de energia relativística, oriunda da Teoria da Relatividade Restrita (ou Especial), para uma partícula de massa $m$

$$
E=m c^{2}=\sqrt{p^{2} c^{2}+m_{0}^{2} c^{4}}
$$

\section{Energia cinética}

Outra maneira, de expressar a energia relativística de uma partícula em movimento, é através da inclusão de ambos os termos de energia de repouso e cinética (associada a este movimento)

$E=E_{0}+T$

ou

$T-m c^{2}-m_{0} c^{2}$

Isto é essencialmente a definição da energia cinética de uma partícula com o excesso de energia, devido à sua massa de repouso. Para velocidades baixas, esta expressão aproxima-se da representação da energia cinética não-relativística, como demonstrado a seguir: ${ }^{15}$ tomando-se a expressão dada pela Equação 16 e usando a Equação 5, a expressão em $T$ torna-se

$T=m_{0} \gamma c^{2}-m_{0} c^{2}$

ou

$$
T=m_{0} c^{2}\left[\frac{1}{\sqrt{1-\frac{v^{2}}{c^{2}}}}-1\right]
$$

Agora expandindo a expressão da raiz quadrada da Equação 18 a partir do teorema binomial

$(a+x)^{n}=\sum_{k=0}^{n} \frac{n !}{(n-k) ! k !} a^{n-k} x^{k}=a^{n}+n a^{n-1} x+\frac{n(n-1)}{2 !} a^{n-2} x^{2}+\cdots$

tem-se

$\left(1-\frac{v^{2}}{c^{2}}\right)^{-1 / 2}=1+\left[\frac{1}{2}\right] \frac{v^{2}}{c^{2}}+\frac{-\frac{1}{2}\left[-\frac{3}{2}\right]}{2 !} \frac{v^{4}}{c^{4}}+\cdots$ 
$T=\frac{1}{2} m_{0} v^{2}+\frac{3}{8} \frac{m_{0} v^{4}}{c^{2}}+\frac{5}{16} \frac{m_{0} v^{6}}{c^{4}}+\cdots$

que para $v \ll c$ dá

$T \approx \frac{1}{2} m_{0} v^{2}$

\section{Aplicação para energia cinética}

Uma das aplicações interessantes, observadas em situações de grandes velocidades, foi a experiência elaborada por Bertozzi. ${ }^{16}$ É um experimento útil para ensinamento de relatividade, enfatizando a existência de uma velocidade limite universal igual à velocidade da luz. O experimento faz uso de um acelerador linear de elétrons e consiste em medir o tempo que os elétrons levam para percorrer determinada distância. Variando-se a voltagem da fonte de elétrons, determina-se a energia cinética que os mesmos possuem. Deste modo, o experimento permite observar como a energia cinética varia com a velocidade. $\mathrm{Na}$ Tabela 1 estão os dados de energia cinética para acelerar os elétrons e dos tempos necessários para eles percorrerem $8,40 \mathrm{~m}$ de caminho no gerador de Van de Graaff. ${ }^{19}$ Na Tabela 2 estão os resultados obtidos a partir destes dados em termos da relação $v^{2} / c^{2}$.

A velocidade $v$ da partícula é calculada pela expressão

$v=\Delta S / \Delta t$

para o espaço percorrido $\Delta S$ de $8,40 \mathrm{~m}$ no intervalo de tempo $\Delta t$ obtido dos dados da $2^{\text {a }}$ coluna da Tabela 1 considerando-se o tempo inicial igual a zero.

A expressão $\left(v^{2} / \mathrm{c}^{2}\right)_{\text {clas }}$, dada a partir do modelo da mecânica clássica newtoniana, é obtida considerando-se que

$$
T=\frac{m_{0} v^{2}}{2} \Rightarrow 2 T=m_{0} v^{2} \Rightarrow \frac{2 T}{c^{2}}=m_{0} \frac{v^{2}}{c^{2}} \Rightarrow\left(\frac{v^{2}}{c^{2}}\right)_{\text {clas }}=\frac{2 T}{m_{0} c^{2}}
$$

Tabela 1. Valores da energia cinética e do tempo necessário para o elétron percorrer a distância de 8,40 $m$ no gerador de Van de Graaff

\begin{tabular}{lc}
\hline $\mathrm{T}(\mathrm{MeV})$ & $\mathrm{t}\left(10^{-8} \mathrm{~s}\right)$ \\
\hline 0,5 & 3,23 \\
1,0 & 3,08 \\
1,5 & 2,92 \\
4,5 & 2,84 \\
15,0 & 2,80 \\
\hline
\end{tabular}

Tabela 2. Valores das relações entre as velocidades do elétron e da luz $-v^{2} / c^{2}$, considerando os dados experimentais e as expressões oriundas da mecânica clássica e contendo efeitos relativísticos ${ }^{(a)}$

\begin{tabular}{lccc}
\hline$v\left(10^{8} \mathrm{~m} / \mathrm{s}\right)^{(\mathrm{b})}$ & $\left(v^{2} / c^{2}\right)_{\exp }$ & $\left(v^{2} / c^{2}\right)_{\text {clas }}{ }^{(\mathrm{c})}$ & $\left(v^{2} / c^{2}\right)_{\text {rel }}^{(\mathrm{d})}$ \\
\hline 2,60 & 0,75 & 1,95 & 0,74 \\
2,73 & 0,83 & 3,90 & 0,86 \\
2,80 & 0,92 & 5,85 & 0,94 \\
2,96 & 0,99 & 17,56 & 0,99 \\
3,00 & 1,00 & 58,54 & 1,00 \\
\hline
\end{tabular}

${ }^{(a)}$ Valores utilizados para as constantes: $c$ (velocidade da luz no vácuo) $=3,00 \times 10^{8} \mathrm{~m} / \mathrm{s} ; e$ (carga do elétron) $=1,60 \times 10^{-19} \mathrm{C} ; m_{0}$ (massa de repouso do elétron) $=9,11 \times 10^{-31} \mathrm{~kg}$; ${ }^{\left({ }^{b}\right)} \mathrm{Eq} .(23) ;{ }^{(\mathrm{c})} \mathrm{Eq} .(24) \mathrm{e}^{(\mathrm{d})}$ Eq. (25).
Já a expressão $\left(v^{2} / \mathrm{c}^{2}{ }_{\text {rel }}\right.$ considera aspectos relativísticos e pode ser obtida rearranjando-se matematicamente a Equação 18.

$T=m_{0} c^{2}\left[\frac{1}{\sqrt{1-\frac{v^{2}}{c^{2}}}}-1\right] \Rightarrow\left(\frac{v^{2}}{c^{2}}\right)_{\text {rel }}=1-\left[\frac{m_{0} c^{2}}{\left(m_{0} c^{2}+T\right)}\right]^{2}$

A dependência da velocidade de uma partícula com sua energia cinética exibe uma velocidade limite e também mostra a relação newtoniana com uma aproximação apropriada às baixas velocidades - Equação 21. Um bom exemplo é considerar a velocidade do elétron igual a $100 \mathrm{~km} / \mathrm{h}(27,78 \mathrm{~m} / \mathrm{s})$, que fornece a relação $\left(\mathrm{v} / \mathrm{c}^{2}\right)$ igual a $8,5910^{-15}$, tornando o termo cinético $m_{0} c^{2}(\gamma-1)$ praticamente nulo, semelhante à energia cinética clássica $-m v^{2} / 2$, igual a $3,5110^{-28} \mathrm{~J}$.

\section{Efeito Compton}

Os trabalhos preliminares efetuados por Compton no campo de raios X permiti que ele desenvolvesse uma teoria sobre a intensidade de reflexão destes raios em cristais, como um meio de estudar o arranjo de elétrons. Este estudo levou-o, em 1922, à descoberta do aumento do comprimento de onda de raios $\mathrm{X}$ devido ao espalhamento da radiação incidente por elétrons livres, o que implicava que os quanta espalhados tinham menos energia do que os quanta incidentes originais, como mostrado na Figura 1. Este efeito ilustra claramente o conceito de partícula para radiação eletromagnética. ${ }^{1} \mathrm{~A}$ análise feita por Compton foi tratar os fótons de raios-X como partículas e aplicar a conservação de energia e de momento para a colisão de um fóton com um elétron estacionário.

Fótons

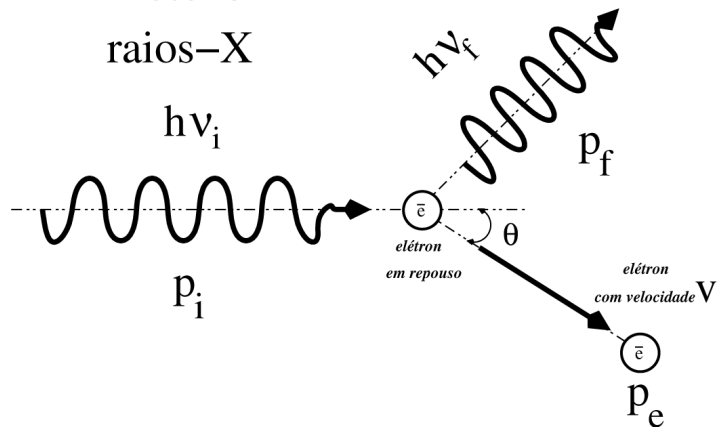

Figura 1. Efeito Compton

\section{Para a conservação de energia}

Pelo princípio da conservação de energia, a energia da radiação eletromagnética incidente (fótons de raios $\mathrm{X}$ de frequiência $v_{\mathrm{i}}$ ) é transferida para o elétron em repouso que adquire velocidade $v$ (energia $T$ ) e para a radiação espalhada de frequiência $v_{f}$. Assim,

$h v_{i}=h v_{f}+T$

A substituição do termo de energia cinética na Equação 26 pela expressão dada pela Equação 16 fornece

$h v_{i}=h v_{f}+m c^{2}-m_{0} c^{2}$

e $m c^{2}$ por $\sqrt{p_{e}^{2} c^{2}+m_{o}^{2} c^{4}}$ - Equação 14 
$h v_{\mathrm{u}}=h v_{f}+\sqrt{p_{e}^{2} c^{2}+m_{o}^{2} c^{4}}-m_{0} c^{2}$

Elevando-se ao quadrado a Equação 28 e rearranjando, tem-se

$p_{e}^{2} c^{2}=\left(h v_{1}-h v_{f}+m_{0} c^{2}\right)^{2}-m_{0}^{2} c^{4}$

Assim,

$$
\begin{aligned}
p_{e}^{2} c^{2} & = & \left(h v_{\mathrm{\imath}}-h v_{f}\right)^{2}+2\left(h v_{\mathrm{\imath}}-h v_{f}\right) m_{0} c^{2}+m_{0}^{2} c^{4}-m_{0}^{2} c^{4} \\
& = & \left(h v_{\mathrm{\imath}}-h v_{f}\right)^{2}+2\left(h v_{\mathrm{\imath}}-h v_{f}\right) m_{0} c^{2}
\end{aligned}
$$

que fornece

$p_{e}^{2} c^{2}=\left(h v_{i}\right)^{2}+\left(h v_{f}\right)^{2}-2 h^{2} v_{i} v_{f}+2 m_{0} c^{2}\left(h v_{i}-h v_{f}\right)$

Já para a conservação do momento a representação é

$\overrightarrow{p_{i}}=\overrightarrow{p_{f}}+\overrightarrow{p_{e}}$

Elevando-se o momento do elétron ao quadrado e colocando-o em termos do produto escalar de vetores, obtém-se

$p_{e}^{2}=\left(\overrightarrow{p_{i}}-\overrightarrow{p_{f}}\right) \cdot\left(\overrightarrow{p_{i}}-\overrightarrow{p_{f}}\right)=p_{i}^{2}+p_{f}^{2}-2 p_{i} p_{t} \cos \theta$

Para o fóton, o uso da expressão relativística do momento,

$p=\frac{m_{0} v}{\sqrt{1-\frac{v^{2}}{c^{2}}}}$

é inadequada, pois como a massa de repouso tende a zero e a velocidade da radiação eletromagnética tende a $c$, tanto o numerador quanto o denominador também tendem a zero, levando a uma indefinição matemática. Assim, para determinar o momento do fóton é preciso utilizar a expressão geral - Equação 14,

$E=m c^{2}=\sqrt{p^{2} c^{2}+m_{0}^{2} c^{4}}$

Considerando-se a massa de repouso do fóton nula, ${ }^{20}$ e por se tratar de radiação eletromagnética, a energia pode ser dada pela Equação de Planck,

$$
p c=h v=\frac{h c}{\lambda} \Rightarrow p=h v \text { ou } p=\frac{h}{\lambda}
$$

que é a relação onda e partícula para a radiação eletromagnética.

Multiplicando-se a Equação 32 por $c^{2}$, fornece

$p_{e}^{2} c^{2}=p_{i}^{2} c^{2}+p_{f}^{2} c^{2}-2 p_{\imath} c p_{f} c \cos \theta$

Os momentos lineares inicial e final dos fótons na Equação 36 são substituídos pela relação $p c=h v$, dada pela Equação 35 , levando à expressão

$p_{e}^{2} c^{2}=\left(h v_{i}\right)^{2}+\left(h v_{f}\right)^{2}-2 h v_{1} h v_{f} \cos \theta$

E, finalmente igualando as Equações 30 e 37, chega-se a

$\left(h v_{i}\right)^{2}+\left(h v_{f}\right)^{2}-2 h^{2} v_{\iota} v_{f}+2 m_{0} c^{2}\left(h v_{i}-h v_{f}\right)=$

$=\left(h v_{i}\right)^{2}+\left(h v_{f}\right)^{2}-2 h v_{\iota} h v_{f} \cos \theta$ obtendo-se

$$
-h v_{\imath} v_{f}+m_{0} c^{2}\left(v_{\iota}-v_{f}\right)=-h v_{\iota} v_{f} \cos \theta
$$

$$
\begin{aligned}
\mathrm{Ou} & \\
-h v_{\iota} v_{f}+m_{0} c^{2}\left(v_{\imath}-v_{f}\right) & =-h v_{\iota} v_{f} \cos \theta \\
m_{0} c^{2}\left(v_{\iota}-v_{f}\right) & =h v_{\iota} v_{f}(1-\cos \theta) \\
m_{0} c^{2}\left(\frac{c}{\lambda_{i}}-\frac{c}{\lambda_{f}}\right) & =\frac{h c^{2}}{\lambda_{i} \lambda_{f}}(1-\cos \theta) \\
m_{0} c\left(\frac{\lambda_{f}-\lambda_{i}}{\lambda_{i} \lambda_{f}}\right) & =\frac{h}{\lambda_{i} \lambda_{f}}(1-\cos \theta)
\end{aligned}
$$

que é a fórmula padrão de Compton

$\lambda_{f}-\lambda_{\mathrm{t}}=\Delta \lambda=\frac{h}{m_{0} c}(1-\cos \theta)$

\section{Relação de De Broglie}

O experimento de Davisson e Germer (Figura 2) demonstrou características de onda para o elétron, de acordo com a hipótese de De Broglie. A relação matemática, que expressa esta hipótese, pode ser obtida considerando-se a expressão da energia relativística dada pela Equação $15 .{ }^{15}$ Ela pode ser expressa reescrevendo-se a componente cinética em termos de $\gamma$ - Equação17,
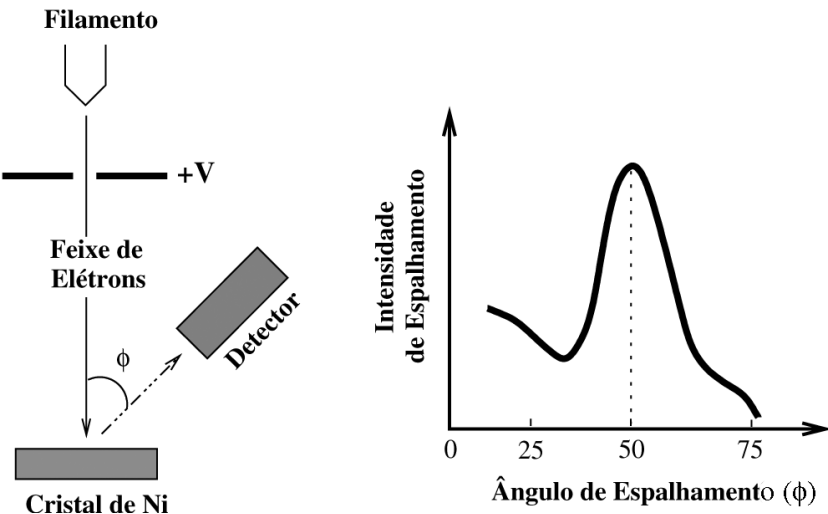

Figura 2. Experimento de Davisson e Germer

$T=m_{0} c^{2}(\gamma-1)$

levando-se a

$E=m_{0} c^{2}+m_{0} c^{2}(\gamma-1)$

ou

$E=m_{0} c^{2} \gamma$

que é essencialmente $E=m c^{2}$, para $m=m_{0} \gamma$.

Substituindo-se agora a expressão do momento relativístico da Equação $6-p=m v=m_{0} v \gamma$, na expressão de energia - Equação 42, tem-se

$E=p \frac{c^{2}}{v}$

Assim, quando $v \rightarrow c$ a partícula se comporta com onda eletromagnética, e deste modo, sua energia passa a ser descrita em termos 
de sua freqüência de onda - Equação de Planck

$$
h v=p \frac{c^{2}}{c} \Rightarrow h \frac{c}{\lambda}=p \frac{c^{2}}{c}
$$

ou

$$
p=\frac{h}{\lambda}
$$

que é a relação onda e partícula para o elétron.

\section{CONCLUSÃO}

A descrição do elétron, sob o ponto de vista de onda ou partícula, é uma das possibilidades de se apresentar o modelo atômico atual. Uma análise mais profunda dos aspectos relacionados a esta descrição de comportamento foi apresentada neste trabalho. Assim, que este material seja um guia de consulta para aqueles que procuram conhecer um tratamento mais rigoroso e distinto daqueles apresentados em muitos livros textos introdutórios de Química, Físico-Química ou de Química Quântica.

\section{AGRADECIMENTOS}

Ao Instituto de Química da UNICAMP, ao Conselho Nacional de Desenvolvimento Científico (CNPq) e à Fundação de Amparo à Pesquisa do Estado de São Paulo (FAPESP), bem como às observações e sugestões feitas pelos assessores, que foram muito pertinentes e enriqueceram o texto final.

\section{REFERÊNCIAS E NOTAS}

1. http://nobelprize.org/nobel_prizes/physics/laureates, acessada em Agosto 2007.

2. Rosa, P. S.; Dissertação de Mestrado, Universidade Estadual de Campinas, Brasil, 2004.
3. Rosa, P. S.; Martins, R. A.; Sci. Am. Brasil 2006, 13, 44.

4. Faria, P.; Estrutura Atômica e Ligação Química, Ed. da UNICAMP: Campinas, $1^{\text {a }}$ ed., 1999, p. 3.

5. Kotz, J. C.; Treichel, P. M.; Química Geral 1 e Reações Químicas, Thomson Learning Ltda: São Paulo, 5 a ed., 2005, p. 241.

6. Brown, T. L.; LeMay, H. E.; Burstein, B. E.; Química, a ciência central, Pearson Prentice Hall: São Paulo, 2005, p. 192.

7. Ball, D. W.; Físico-Química, Thomson Learning Ltda: São Paulo, 2005, vol. 1, p. 267.

8. Atkins, P. W.; Physical Chemistry, Oxford University Press: Oxford, $5^{\text {th }}$ ed., 1994, p. 367-368.

9. McQuarrie, D. A.; Simon, J. D.; Physical Chemistry - A Molecular Approach, University Science Books: Sausalito, 1997, p. 15.

10. Dias, J. J. C. T.; Química Quântica - Fundamentos e Métodos, Fundação Calouste Gulbenkian: Lisboa, 1980, p. 15.

11. Levine, I. N.; Quantum Chemistry, Prentice Hall: New York, $4^{\text {th }}$ ed., 1991, p. 5.

12. Braga, J. P.; Fundamentos de Química Quântica, Editora UFV: Viçosa, 2007, p. 46.

13. Feynman, R. P.; Leighton, R. B.; Sands, M.; The Feynman Lectures on Physics, Addison-Wesley Pub. Company, Inc: California, 1964, cap. 38.

14. Alcácer, L.; Introdução à Química Quântica Computacional, IST Press Instituto Superior Técnico: Lisboa, 2006, p. 23-33.

15. http://hyperphysics.phyastr.gsu.edu/hbase/hframe.html, acessada em Março 2007.

16. Bertozzi, W.; Am. J. Physics 1964, 32, 551.

17. Embora o momento linear seja uma grandeza vetorial, por questão de simplicidade a representação de $\vec{p}$ referirá ao módulo de $p$, assim como para outras grandezas de mesma natureza.

18. Um bom exercìcio é desenvolver a Eq.(13) diretamente a partir da definição de massa $-m=m_{0} \gamma$, embora seja um procedimento menos elegante do que o apresentado é bem mais simples.

19. Vide ref. 12 para mais detalhes do experimento.

20. Efetivamente é não nula, mas muito pequena. 